\title{
Artrodese interfalângica: eficácia e retorno laboral com fios Kirschner e parafuso Acutrak ®
}

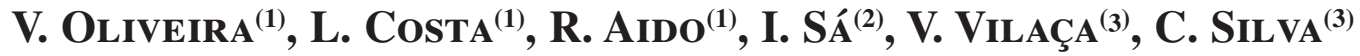 \\ (1) Interno Complementar de Ortopedia, Centro Hospitalar do Porto, Portugal \\ (2) Interna Complementar de Cirurgia Plástica e Reconstructiva, Hospital de São João - Porto, \\ PORTUGAL \\ (3) Assistente Hospitalar, Centro Hospitalar do Porto, Portugal
}

Correspondência:

Dra. Vânia Oliveira

Centro Hospitalar do Porto - Hospital de Santo António

Serviço de Ortopedia e Traumatologia

Largo Prof. Abel Salazar

4099-001 Porto - Portugal

Telef.: 00351918151915

e-mail: vaniacoliveira@gmail.com

Conflito de interesses:

Os autores declaram que não têm nenhum conflito de interesses.

A artrodese interfalângica (IF) é um procedimento osteoarticular frequente e os fios de Kirschner são os mais usados. Outras técnicas descritas são parafusos compressivos ou, recentemente, parafuso sem cabeça, completamente roscado, Acutrak ${ }^{\circledR}$ (Acumed LLC, Hillsboro, OR). Os autores propuseram comparar duas técnicas de artrodese IF: com fios Kirschner e parafusos Acutrak®. Trata-se de um estudo retrospectivo de 2009 a 2012 englobando 67 artrodeses. Destes foram excluídos doentes com infecção, osteopenia ou perda óssea como artrite reumatóide. Obteve-se um total de 53 artrodeses: 22 com fios Kirschner (grupo I) e 31 com parafusos Acutrak $\AA$ (grupo II). Foi determinada a taxa e tempo de fusão, complicações e tempo de retorno laboral. Os resultados foram analisados pelo SPSS® $20 \mathrm{Sta}$ tistics $(p<0.05)$. No grupo I, verificou-se $77.3 \%$ de fusão com $9 \%$ de complicações menores. No grupo II ocorreu consolidação em 100\%, na ausência de complicações. O tempo médio de fusão foi 9.6 (r: 6-14) semanas no grupo I e 5.1 (r: 4-8) semanas no grupo II. O retorno laboral foi às 11.5 semanas e 5.4 semanas, respectivamente. O parafuso Acutrak ${ }^{\circledR}$
Interphalangeal (IP) arthrodesis is a frequent procedure and the Kirschner-wires are frequently used. Others are compressive screws or, recently, a headless screw, Acutrak® (Acumed LLC, Hillsboro, OR). The authrors proposed to compare 2 different techniques of IF arthrodesis: Kirschner-wires and Acutrak ${ }^{\circledR}$ screw. Retrospectively were analysed 67 arthrodesis between 2009 and 2012. Patients with infection, osteopenia and bone defects as rheumatoid arthritis were eliminated. A total of 53 arthrodesis were included: 22 Kirschner-wires (group I) and 31 Acutrak ${ }^{\circledR}$ screw (group II). Fusion rate and time, complications and time to return to work were determinate in all patients. Results were statistically evaluated by SPSS ${ }^{\circledR} 20$ Statistics $(\mathrm{p}<0.05)$. Fusion occurred in $77.3 \%$ of group I with $9 \%$ of minor complications. The group II presented fusion in $100 \%$, without complications. The mean time to fusion was 9.6 (r: 6-14) weeks on group I, and 5.1 (r: 4-8) weeks on group II. Patients were able to return to work at 11.5 weeks and 5.4 weeks, respectively. The Acutrak ${ }^{\circledR}$ presents a more efficient arthrodesis than the Kirschner-wires and an early return to work. 
permite uma artrodese mais eficaz que os fios Kirschner e retorno laboral precoce.

Palavras-chave: artrodese interfalângica, parafuso sem cabeça, Acutrak ${ }^{\circledR}$, fios Kirschner, consolidação, eficácia, retorno laboral.
Key Words: interphalangeal arthrodesis, headless screw, Acutrak $®$, Kirschner-wires, fusion, efficacy, return to work.

Rev. Iberam. Cir. Mano - Vol. 39•Núm. 2 • Noviembre 2011 (51-56)

\section{INTRODUÇÃO}

A artrodese interfalângica (IF) é um dos procedimentos osteoarticulares frequentes na mão, indicada para tratar dor e deformidade em situações clínicas como deformidade pós-traumática, reumática, paralítica, congénita, artrítica, infecciosa ou outra como Dupuytren.

Entre as diversas técnicas cirúrgicas de fixação a mais usada recorre a fios de Kirschner. Outras técnicas previamente descritas são os parafusos compressivos e, mais recentemente, parafuso sem cabeça, completamente roscado, cónico, com passos de rosca de diferentes tamanhos e auto-tradante, Acutrak ${ }^{\circledR}$ (Acumed LLC, Hillsboro, OR). A decisão da técnica cirúrgica depende da avaliação de osteopenia e perda óssea.

$\mathrm{Na}$ literatura, as técnicas associadas a maior rigidez estrutural e compressão apresentam resultados superiores devido à estabilidade atingi$\mathrm{da}^{1-4}$. Leibovic concluiu que a compressão acelera a fusão de articulações menores ${ }^{1}$.

Os fios Kirschner fornecem rigidez estrutural mas apresentam consistentemente elevadas taxas de complicações como $42 \%$ no estudo de Stern e Fulton $^{2}$, englobando 4\% de infecção, $15 \%$ de necrose cutânea e $11 \%$ de pseudartrose. Em outros estudos como o de Leibovic ${ }^{1}$ a taxa de pseudartrose atinge os $21 \%$.

Os parafusos auto-compressivos permitem atingir elevada compressão e estabilidade e o parafuso Acutrak ${ }^{\circledR}$ apresenta vantagens teóricas. Além disso, ao contrário dos fios Kirschner, têm fixação e ancoragem intra-óssea. Na literatura, a taxa de consolidação do Acutrak ${ }^{\circledR}$ varia entre $100 \%$ no estudo de Song et $\mathrm{al}^{3}$ e $85 \%$ publica- do por Brutus et $\mathrm{al}^{4}$, associado a reduzida taxa de complicações de $4.3 \%$ (1 caso de fractura da cortical dorsal da falange proximal do polegar) no primeiro estudo ou de $10 \%$ de lesão do leito ungueal e $10 \%$ de infecção, no segundo estudo. Rehak $^{5}$ reforça o curto tempo de imobilização e está generalizado o tempo de fusão entre 4-8 semanas $^{1,3-5}$.

Os autores propuseram comparar 2 técnicas de artrodese IF: fios Kirschner e parafuso Acutrak ${ }^{2}$.

\section{DOENTES E MÉTODO}

Foram analisados retrospectivamente 49 doentes, num total de 67 artrodeses IF entre janeiro de 2009 e abril 2012: 31 com fios Kirschner (grupo I) e 36 com micro ou mini-Acutrak ${ }^{\circledR}$ (grupo II). Todas as artrodeses foram realizadas pelo mesmo cirurgião sénior.

TABELA I - Distribuição POR ETIOLOGIA DO GRUPO I (FIOS KIRSCHNER) E GRUPO II (PARAFUso ACUTRAK®)

\begin{tabular}{|l|c|c|}
\hline & Grupo I & Grupo II \\
\hline Artrite Traumática & 18 & 12 \\
\hline Artrite Reumática & 6 & 2 \\
\hline Artrite Psoriática & 1 & 3 \\
\hline Osteoartrite $1^{\text {a }}$ & 2 & 4 \\
\hline Paralítica & 1 & 8 \\
\hline Congénita & - & 2 \\
\hline D. Dupuytren & 1 & 5 \\
\hline Infecção Crónica & 2 & - \\
\hline Total & $\mathbf{3 1}$ & $\mathbf{3 6}$ \\
\hline
\end{tabular}



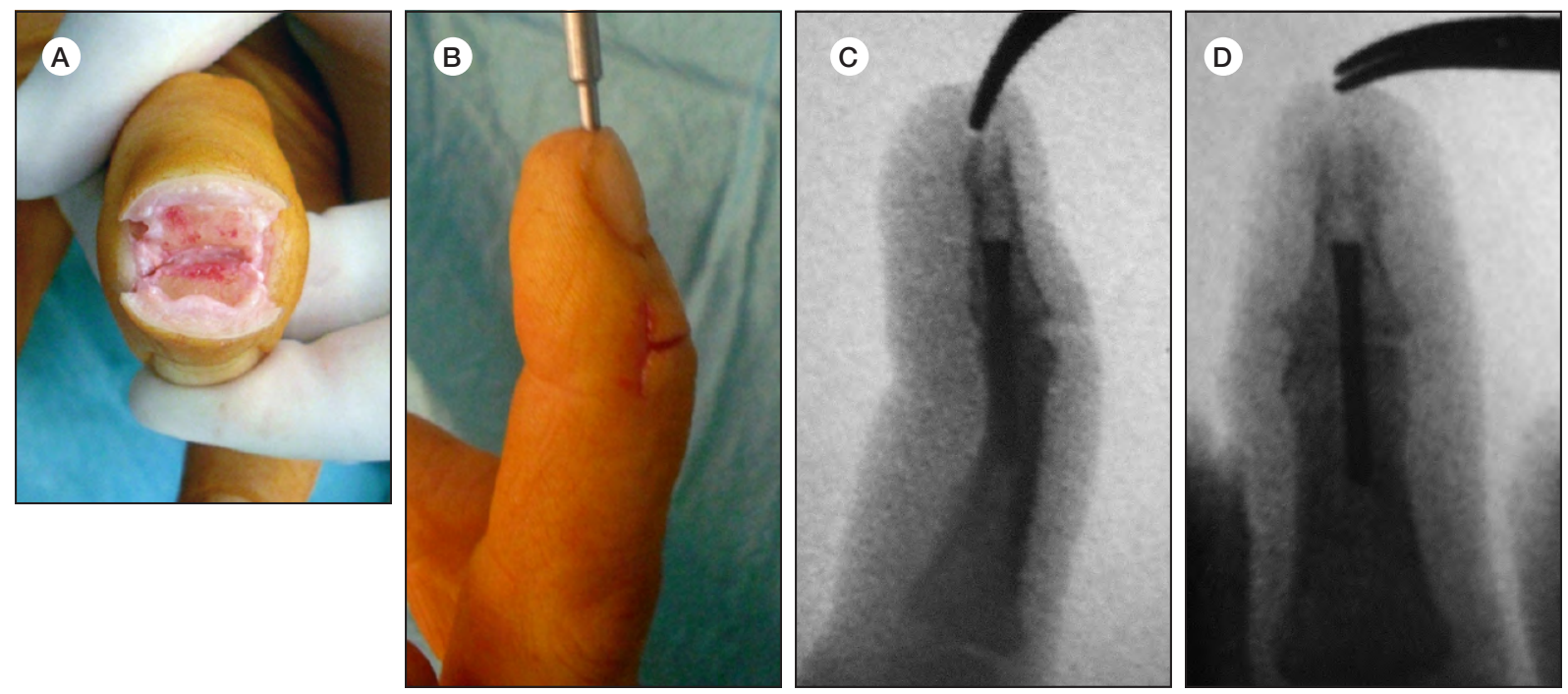

Figura 1. A-B) Imagen per-operatória da técnica de artrodese IFD do $3^{\circ}$ dedo direito com parafuso Acutrak®. Em destaque a osteotomia e posição de fixação. C-D) Rx pós-operatórios. A fusão e retorno laboral ocorreram às 7 semanas.

Ambos os grupos incluíram doentes com todas as etiologias previamente descritas (Tabela I), no entanto, doentes com infecção, artrite reumática e psoriática, com perda óssea ou osteopenia significativas, foram maioritariamente incluídos no grupo I. Isto porque, apesar de inicialmente doentes osteopénicos ou com perda óssea como artrite reumatoide terem sido incluídos no grupo II, apresentaram sistematicamente complicações, nomeadamente pseudartrose e fractura da cortical dorsal da falange proximal do polegar, em 2 casos de artrite reumática.
Além disso, quando infecção o cirurgião opta por fios Kirschner.

Assim, após exclusão dessas etiologias, em ambos os grupos, foram incluídas no estudo um total de 53 artrodeses: 22 do grupo I e 31 do grupo II.

Todos os doentes foram submetidos a uma avaliação clínica e imagiológica com radiografias de face e perfil. A posição de fixação foi entre $30^{\circ}-50^{\circ}$ na articulação IF proximal (IFP), de acordo com o dedo ${ }^{6}$, e entre $0^{\circ}-10^{\circ}$ na articulação IF distal (IFD).

\section{Tabela II - Resultados descritivos}

\begin{tabular}{|l|c|c|c|}
\cline { 2 - 4 } \multicolumn{1}{c|}{} & Grupo I & Grupo II & p-value \\
\hline Doentes & 22 & 31 & \\
\hline Masculino & $13(59 \%)$ & $14(54.8 \%)$ & \\
Feminino & $9(41 \%)$ & $17(45.2)$ & \\
\hline Idade & $42.9(13-68, \pm 12.2)$ & $47.0(15-66, \pm 14.3)$ & 0.28 \\
\hline FU & $22.05(1-40, \pm 13.49$ & $14.77(1-31, \pm 10.3)$ & 0.02 \\
\hline Consolidação & $17(77.3 \%)$ & $31(100 \%)$ & 0.9 \\
\hline Tempo & $9.6(6-14, \pm 2.4)$ & $5.1(4-8, \pm 1.1)$ & \\
\hline Pseudartrose & $5(22.7 \%)$ & 0 & 0.9 \\
\hline Retorno Laboral & $11.5(6-17, \pm 3.0)$ & $5.4(4-8, \pm 1.2)$ & 1.0 \\
\hline Complicações & $2(9 \%)$ & 0 & \\
\hline
\end{tabular}



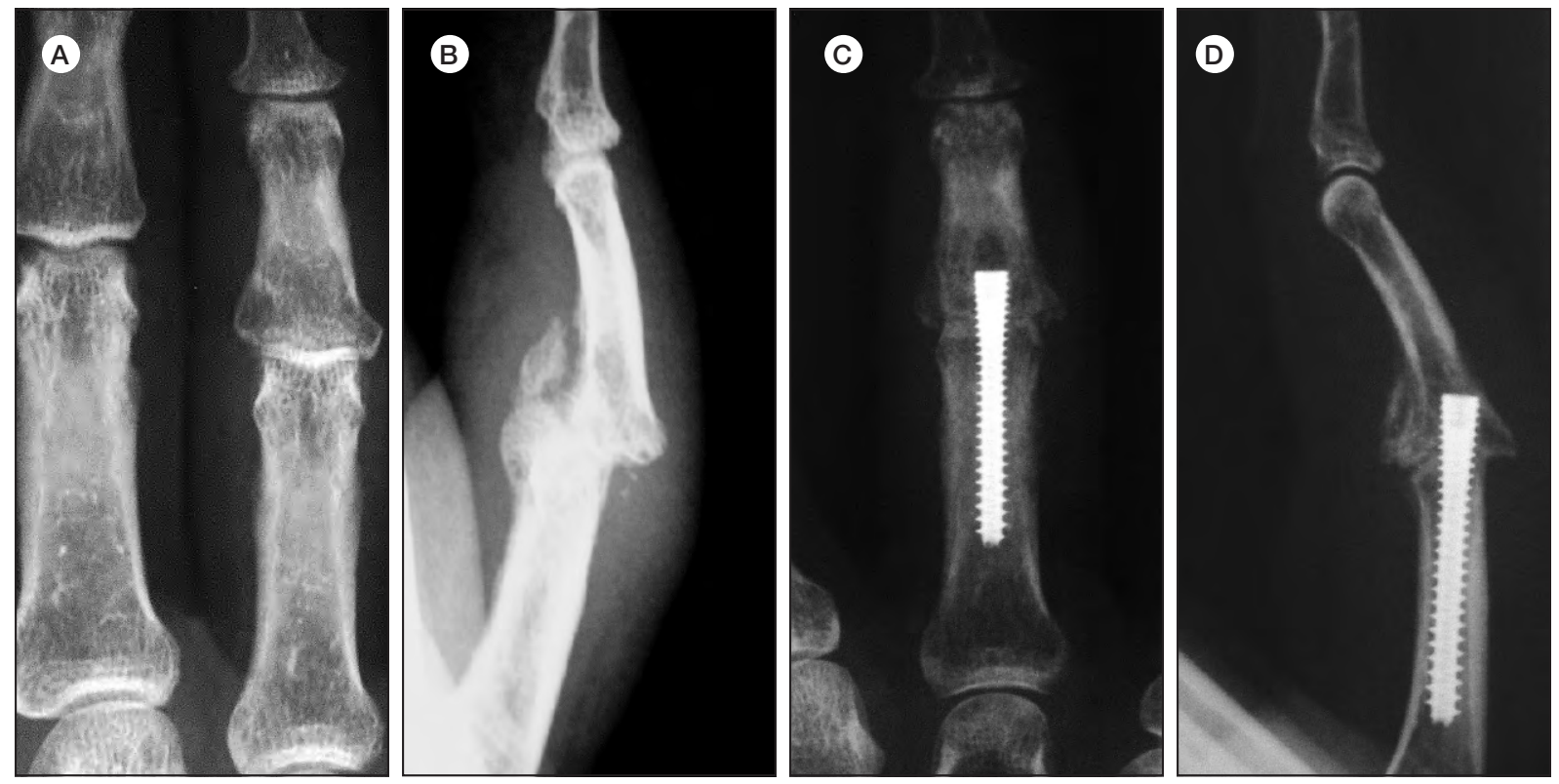

Figura 2. A-B) Rx de face e perfil pre-operatório de doente do sexo masculino de 55 anos com história traumática do qual

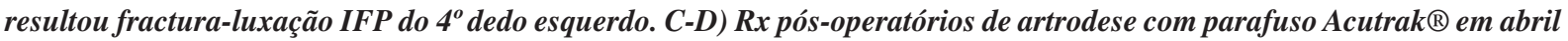
2011. Consolidou e retomou o trabalho às 4 semanas.

Foram avaliados os seguintes parâmetros: consolidação e tempo, complicações, tempo de retorno laboral ou a atividades prévias.

Os resultados foram estatisticamente analisados com SPSS® 20 Statistics software (IBM Chicago-IL, EUA), Chi-Square e Fisher's Tests, com $p<0.05$.

\section{RESULTADOS}

Os resultados descritivos estão presentes na Tabela II. Ambos os grupos apresentaram características semelhantes com predomínio do sexo feminino e maioritariamente atingimento do lado esquerdo. O grupo I apresentou seguimento médio de 24.8 (r: 1-40) meses e o grupo II 15.7 (r: 1-31) meses. A idade média foi 45.7 (r: 13-74) anos e 49.8 (r: 15-75) anos, respectivamente.

A consolidação verificou-se em $77.3 \%$ do grupo I e 100\% do grupo II (Figuras 1, 2), e o tempo médio de fusão foi 9.6 (r: 6-14) e 5.1 (r: 4-8) semanas, respectivamente. Ocorreu pseudartrose assintomática em 5 casos de artrite pós-traumática do grupo I (Figura 3). O tempo médio de retorno laboral ou a atividades prévias foi 11.5 (r: 6-17) semanas no grupo I e 5.4 (r: 4-8) semanas no grupo II.
Apenas foram registadas complicações menores. No grupo I ocorreram 2 casos de infecção superficial (9\%), em 2 doentes com artrite póstraumática, que responderam a antibioterapia. No grupo II não foram registadas complicações.

Comparativamente, o grupo Acutrak ${ }^{\circledR}$ apresenta uma artrodese significativamente mais eficaz $(\mathrm{p}=0.02)$, com tendência a reduzidas complicações $(\mathrm{p}=1.0)$, curto tempo de imobilização $(\mathrm{p}=0.9)$ e retorno laboral precoce $(\mathrm{p}=0.9)$.

Além disso, no grupo II a consolidação associa-se significativamente a menor imobilização e retorno laboral mais rápido $(\mathrm{p}=0.002)$. A diferença no seguimento dos grupos não é estatisticamente significativa $(\mathrm{p}=0.28)$, nem interferiria com os parâmetros avaliados.

\section{DISCUSSÃO}

A artrodese IF reduz a mobilidade mas a estabilidade que alcança permite alívio da dor e melhoria da função da mão ${ }^{1-10,12}$, sendo do ponto de vista estético perfeitamente aceitável.

Este estudo apresenta as limitações inerentes a ser retrospectivo e com reduzida amostra. Os fios Kirschner são economicamente mais vantajosos mas neste estudo revelam maior taxa de 


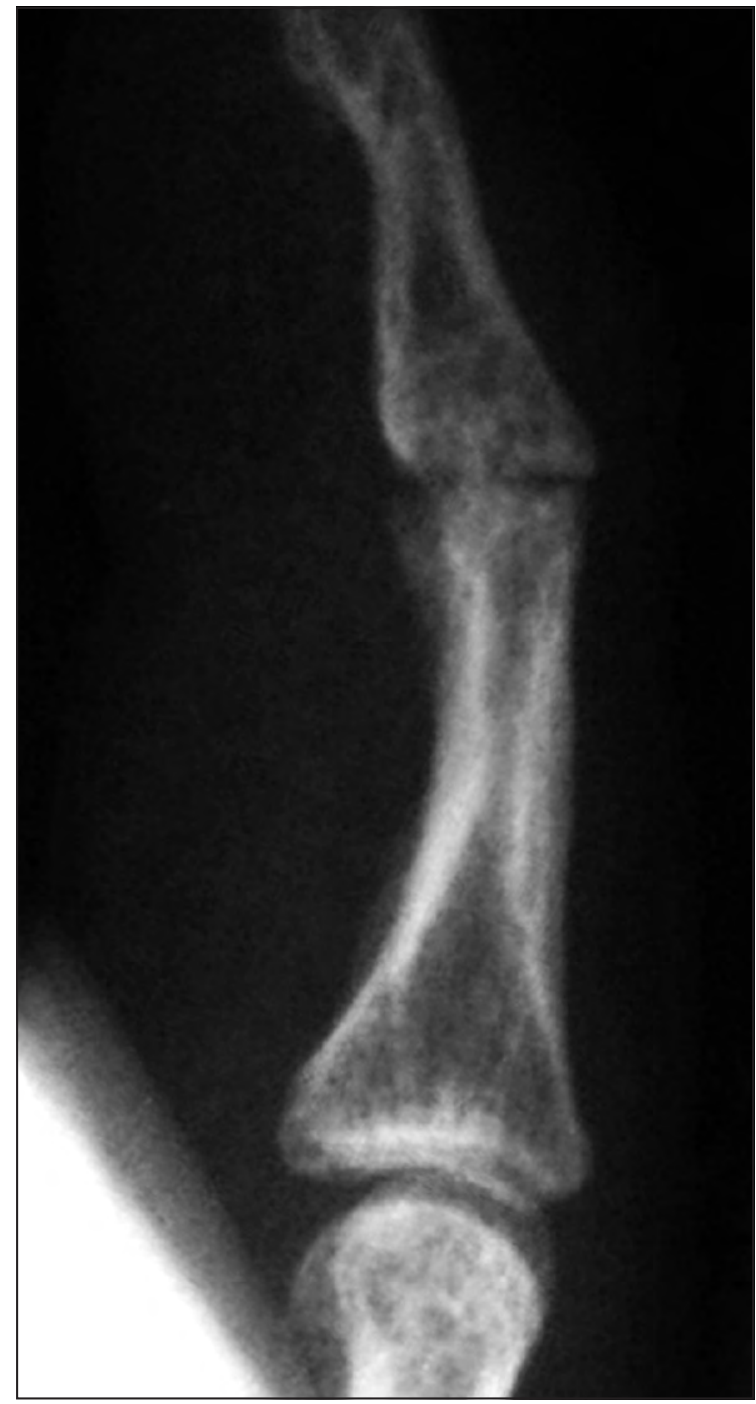

Figura 3. Tratamento de artrite pós-traumática. $O R x$ de perfil mostra estado de pseudartrose pós-artrodese IFD do $5^{\circ}$ dedo da mão direita com fios Kirschner. $O$ doente encontra-se assintomático e retomou o trabalho às 17 semanas.

complicações e pseudartrose, o que vai de encontro a outros estudos previamente publicados ${ }^{1,2}$. Comparativamente, neste estudo o Acutrak ${ }^{\circledR}$ demonstra elevada taxa de consolidação sendo que esta ocorre em todos os doentes até às 8 semanas. Paralelamente, não apresenta complicações e permite um rápido retorno laboral ou a atividades prévias, entre as 4-8 semanas, também de acordo com a literatura.

Apesar de as publicações nesta área serem reduzidas, em geral, o parafuso Acutrak $®$ apresenta elevada taxa de fusão com menor imobilização e reduzidas complicações. Apresenta-se, inclusi- ve, eficaz mesmo em casos de luxação prévia, ou alguma perda óssea, devido à estabilidade que alcança. Além disso, o Micro-Acutrak® mostrase adequado para a articulação IFD não tendo sido registadas complicações comparativamente com outras técnicas cirúrgicas ${ }^{2,7}$.

Sabe-se que as complicações associadas a artrodese IF podem englobar pseudartrose, infecção, necrose de pele, fractura cortical dorsal de falange e lesão do leito ungueal ${ }^{2,4,7}$.

A experiência do cirurgião também contribuiu certamente para estes resultados.

Salienta-se que a artrodese é efetiva, segura e reprodutível no tratamento da artrite nas articulações IF e metacarpofalângica ${ }^{8}$ e os parafusos de compressão têm mostrado superioridade em relação aos fios Kirschner ${ }^{9}$. Estudos prévios demonstram que o parafuso compressivo $\mathrm{HCS}^{7}{ }^{7}$ ou o implante intramedular elástico X-Fuse ${ }^{\circledR 10}$, também permitem compressão adequada mas devido ao menor diâmetro central apresentam alguma oscilação. Gausepohl et al ${ }^{11}$ concluem que um diâmetro de rosca superior está associado a maior poder de fixação do parafuso e isto deve-se em parte ao efeito de impactação entre as roscas e a estrutura de osso esponjoso. O parafuso Acutrak ${ }^{\circledR}$ apresenta uma forma cónica com passo de rosca de diferentes tamanhos, sendo maior distalmente, de forma a contribuir para o efeito de impactação, compressão e estabilidade. Além disso, o Acutrak ${ }^{\circledR}$ pelo facto de ser completamente roscado confere maior estabilidade à montagem minimizando a perda de posição inicial. Por outro lado, o Acutrak ${ }^{\circledR}$ não necessita remoção, ao contrário dos fios Kirschner que são removidos e, por vezes, implicam nova ida ao bloco operatório para extração sob anestesia.

No doente osteopénico a osteólise é uma complicação frequente levando à desmontagem do sistema. Nestes casos o parafuso Acutrak® perde efeito de impactação e os fios Kirschner apresentam vantagens não apenas económicas mas também pelo facto de terem fixação cortical.

\section{CONCLUSÃO}

Neste estudo o parafuso Acutrak® é superior ou tendencialmente superior em todos os parâmetros avaliados, mas é necessário selecionar 
doentes de forma a optimizar os resultados. Assim, os fios Kirschner são a opção preferencial dos autores quando existe infecção, artrite reumática, perda óssea ou osteopenia. Em todas as restantes etiologias, o parafuso Acutrak ${ }^{\circledR}$ permite uma artrodese IF significativamente mais efetiva que os fios Kirschner, com menores complicações e retorno laboral mais precoce.

\section{BIBLIOGRAFIA}

1. Leibovic SJ. Instructional Course Lecture: Arthrodesis of the interphalangeal joints with headless compression screws. $J$ Hand Surg Am, 2007; 32: 1113-9.

2. Stern PJ, Fulton DB. Distal interphalangeal joint arthrodesis: an analysis of complications. J Hand Surg Am, 1992; 17: 113945.

3. Song JH, Lee JY, Chung YG, Park IJ. Distal interphalangeal joint arthrodesis with a headless compression screw: morphometric and functional analyses. Arch Orthop Trauma Surg, 2012; 132: 663-9.

4. Brutus JP, Palmer AK, Mosher JF, Harley BJ, Loftus JB. Use of a headless compressive screw for distal interphalangeal joint arthrodesis in digits: clinical outcome and review of compli- cations. J Hand Surg Am, 2006; 31: 85-9.

5. Rehak DC. Arthrodesis of the distal interphalangeal joint using a headless screw. Oper Tech Orthop, 2007; 17: 140-7.

6. Domalain M, Evans PJ, Seitz WH, Li ZM. Influence of index finger proximal interphalangeal joint arthrodesis on precision pinch kinematics. J Hand Surg Am, 2011; 36: 1944-9.

7. Mantovani G, Fukushima WY, Cho AB, Aita MA, Lino W, Faria FN. Alternative to the distal interphalangeal joint arthrodesis: lateral approach and plate fixation. J Hand Surg Am, 2008; 33: 31-4.

8. Shin EK, Osterman AL. Treatment of thumb metacarpophalangeal and interphalangeal joint arthritis. Hand Clin, 2008; 24: 239-50.
9. Engel J, Tsur H, Farin I. A comparison between $\mathrm{K}$-wire and compression screw fixation after arthrodesis of the distal interphalangeal joint. Plast Reconstr Surg, 1977; 10: 611-4.

10. Savvidou C, Kutz J. Interphalangeal and thumb metacarpophalangeal arthrodesis with an intramedullary implant. Ann Plast Surg, 2013; 70: 34-7.

11. Gausepohl T, Moehring R, Pennig $\mathrm{D}$, Koebke J. Fine thread versus coarse thread: a comparison of the maximum holding power. Injury, 2001; 32 (Suppl): 1-7.

12. Shin AY, Amadio PC. Stiff Finger Joints. Em: Green DP, Hotchkiss RN, Pederson WC, Wolfe SW (Eds). Green's Operative Hand Surgery. 5th ed. Philadelphia: Elsevier Churchill Livingstone, 2005: 417-59. 